\section{Determinants of therapy switch in multiple sclerosis treatment-naïve patients: A real-life study}

Francesco Saccà, Roberta Lanzillo, Alessio Signori, Giorgia T Maniscalco, Elisabetta Signoriello. Salvatore Lo Fermo, Annamaria Repice, Pietro Annovazzi, Damiano Baroncini ${ }^{i D}$, Marinella Clerico, Eleonora Binello, Raffaella Cerqua, Giorgia Mataluni, Simona Bonavita, Luigi Lavorgna, Ignazio Roberto Zarbo, Alice Laroni, Silvia Rossi, Lorena Pareja Gutierrez, Sara La Gioia, Barbara Frigeni, Valeria Barcella, Jessica Frau, Eleonora Cocco, Giuseppe Fenu, Valentina Torri Clerici, Arianna Sartori, Sarah Rasia, Cinzia Cordioli, Alessia Di Sapio, Simona Pontecorvo, Roberta Grasso, Caterina Barrilà, Cinzia Valeria Russo, Sabrina Esposito, Domenico Ippolito, Francesca Bovis, Fabio Gallo and Maria Pia Sormani; On behalf of the iMUST Group

\section{Abstract}

Background: With many options now available, first therapy choice is challenging in multiple sclerosis (MS) and depends mainly on neurologist and patient preferences.

Objectives: To identify prognostic factors for early switch after first therapy choice.

Methods: Newly diagnosed relapsing-remitting MS patients from 24 Italian centers were included. We evaluated the association of baseline demographics, clinical, and magnetic resonance imaging (MRI) data to the switch probability for lack of efficacy or intolerance/safety with a multivariate Cox analysis and estimated switch rates by competing risks models.

Results: We enrolled 3025 patients. The overall switch frequency was $48 \%$ after 3 years. Switch risk for lack of efficacy was lower with fingolimod (hazard ratio $(\mathrm{HR})=0.50 ; p=0.009)$, natalizumab $(\mathrm{HR}=0.13$; $p<0.001)$, dimethyl-fumarate $(\mathrm{HR}=0.60 ; p=0.037)$, teriflunomide $(\mathrm{HR}=0.21 ; p=0.031)$ as compared to interferons. Younger age $(\mathrm{HR}=0.96 ; p<0.001)$, diagnosis delay $(\mathrm{HR}=1.23 ; p=0.021)$, higher baseline Expanded Disability Status Scale $(\mathrm{HR}=1.17 ; p=0.001)$, and spinal cord lesions $(\mathrm{HR}=1.46 ; p=0.001)$ were independently associated with higher inefficacy switch rates. We found lower switch for intolerance/safety with glatiramer acetate $(\mathrm{HR}=0.61 ; p=0.001)$, fingolimod $(\mathrm{HR}=0.35 ; p=0.002)$, and dimethyl-fumarate ( $\mathrm{HR}=0.57 ; p=0.022)$ as compared to interferons, while it increased with natalizumab $(\mathrm{HR}=1.43 ; p=0.022)$. Comorbidities were associated with intolerance switch $(\mathrm{HR}=1.28 ; p=0.047)$.

Conclusion: Several factors are associated with higher switch risk in patients starting a first-line therapy and could be integrated in the decision-making process of first treatment choice.

Keywords: Switch, naïve, persistence, disease modifying therapies, relapsing-remitting, real-life

Date received: 30 March 2018; revised: 25 June 2018; accepted: 27 June 2018

\section{Introduction}

In recent years, therapeutic options for the relapsingremitting (RR) course of multiple sclerosis (RR-MS) have largely increased. The European Medicines Agency (EMA) and the Italian regulatory agency $(\mathrm{AIFA})^{1,2}$ have classified disease modifying therapies
(DMTs) as first- or second-line therapy, according to the risk/benefit profile found in clinical trials.

Since there are no clear predictors of efficacy based on patients' baseline characteristics, first therapy choice depends mainly on personal attitude toward single
Multiple Sclerosis Journal

$1-10$

DOI: $10.1177 /$

1352458518790390

(C) The Author(s), 2018. Reprints and permissions: http://www.sagepub.co.uk/ journalsPermissions.nav
Correspondence to: MP Sormani Department of Health Sciences (DISSAL), Section of Biostatistics, University

of Genova, Via Pastore, 1 , 16132 Genova, Italy. mariapia.sormani@unige.it

Francesco Saccà Roberta Lanzillo Cinzia Valeria Russo Multiple Sclerosis Center, Department of Neuroscience, Reproductive Sciences and Odontostomatology, Federico II University, Naples, Italy

Alessio Signori Francesca Bovis Fabio Gallo

Maria Pia Sormani Department of Health Sciences (DISSAL), Section of Biostatistics, University of Genova, Genova, Italy

Giorgia T Maniscalco Neurological Clinic and Multiple Sclerosis Center, "AORN A.Cardarelli," Naples, Italy

Elisabetta Signoriello Multiple Sclerosis Center, II Division of Neurology, University of Campania Luig Vanvitelli, Naples, Italy

Salvatore Lo Fermo Neurological Clinic, A.O.U. Policlinico Vittorio Emanuele, Catania, Italy

Annamaria Repice 2nd Neurology Unit and CRRSM (Regional Referra Multiple Sclerosis Center), Careggi University Hospital and University of Florence, Florence, Italy

Pietro Annovazzi Damiano Baroncini Multiple Sclerosis Study Center, ASST Valle Olona, PO di Gallarate (VA), Gallarate, Italy 
Marinella Clerico

Clinical and Biological

Sciences Department,

Neurology Unit, University

of Torino, San Luigi

Gonzaga University Hospital,

Torino, Italy

Eleonora Binello

Centro Sclerosi Multipla,

Dipartimento di

Neuroscienze, AOU Città

della Salute e della Scienza di

Torino, Torino, Italy

Raffaella Cerqua

Neurological Clinic,

Department of Experimental

and Clinical Medicine,

Marche Polytechnic

University, Ancona, Italy

Giorgia Mataluni

Policlinico Tor Vergata

Rome, Italy

Simona Bonavita

Luigi Lavorgna

Sabrina Esposito

Domenico Ippolito

Department of Medical,

Surgical, Neurological,

Metabolic and Aging

Sciences, University of

Campania Luigi

Vanvitelli, Naples,

Italy

Ignazio Roberto Zarbo

Department of Clinical and

Experimental Medicine,

University of Sassari, Sassari,

Italy

Alice Laroni

Department of

Neurosciences,

Rehabilitation,

Ophthalmology, Genetics,

Maternal and Child Health

and Center of Excellence

for Biomedical Research

(CEBR) and IRCCS

San Martino-IST, University

of Genova, Genova,

Italy

Silvia Rossi

Lorena Pareja Gutierrez

Valentina Torri Clerici

Neuro-immunology and

Neuromuscolar Diseases

Unit, IRCCS Foundation

Carlo Besta Neurological

Institute, Milan, Italy

Sara La Gioia

Barbara Frigeni

Valeria Barcella

Centro Sclerosi Multipla,

ASST Papa Giovanni XXIII

di Bergamo, Bergamo,

Italy

Jessica Frau

Eleonora Cocco

Giuseppe Fenu

Department of Medical

Sciences and Public

Health, University of

Cagliari, Cagliari,

Arianna Sartori

Neurology Clinic,

Department of Medical,

Surgical, and Health

Sciences, University of

Trieste, Trieste, Italy
DMTs, patients' preferences, and co-existing comorbidities. ${ }^{3,4}$ Recently, new oral compounds have been licensed, ${ }^{5}$ making the choice even more complex.

For highly active RR-MS at onset of disease, with clinical and radiological signs of inflammation, second-line therapies are also considered as first treatment choice. This category has enlarged with alemtuzumab ${ }^{6}$ and more recently ocrelizumab, ${ }^{7}$ in addition to natalizumab and fingolimod. In more aggressive diseases, there are no clear indications regarding the first treatment choice, since real data comparisons deal with the oldest compounds, and mainly with patients switching from other therapies. ${ }^{8-14}$

The scenario of RR-MS treatment is also complicated by the high expectations that neurologists and patients have since the criteria of no evidence of disease activity (NEDA) (absence of relapses, disability increase, and disease activity on magnetic resonance imaging (MRI)) have been introduced..$^{15}$ Patients with poor disease control are switched to different DMTs either with well-recognized higher efficacy or with different mechanisms of action. ${ }^{16}$ This attitude, however, has not been extensively analyzed in terms of efficiency, apart from single-center reports. ${ }^{17-19}$ Side effects may affect patients' tolerance and adherence, ${ }^{20,21}$ so that switches for side effects or poor tolerability are also very frequent. ${ }^{22}$

There are still no established prognostic factors predicting persistence to a given therapy,,$^{23,24}$ so that reallife data can be helpful to identify clinical and demographic characteristics predicting early switch risk. Aims of our study were (1) to provide a snapshot of the prescribing attitude in newly diagnosed Italian MS patients from 2010 through 2017; (2) to describe the switch patterns from first therapy; (3) to define whether baseline characteristics could predict patients' persistence on therapies, guiding first treatment choice.

\section{Patients and methods}

\section{Study design}

We designed a multicenter, retrospective study, involving 24 Italian MS centers. The ethics committee of the coordinating center (Genova) approved the study. Raw data collection was approved by the local ethics committees at all centers. All the centers involved in the study asked for written permission of the use of anonymized personal clinical data for research purposes, and written informed consent was obtained from all study patients included in this study. Inclusion criteria were age over 16 years, diagnosis of
RR-MS (2001 International Panel Diagnostic Criteria and the 2010 revision), ${ }^{25,26}$ and initiating a DMT between January 2010 and June 2017. There were no exclusion criteria.

We collected data using local databases that served as source data. Smaller centers reviewed patient's charts directly. We shared a common database template with predefined criteria for data categorization. All files were merged in one common database by a data manager (A.S.), and further processed for data cleaning and analysis.

We collected demographics (age, gender, level of education) and clinical data at the time of diagnosis (baseline): date of disease onset and diagnosis, relapses in the previous year (excluding relapses leading to diagnostic procedures), Expanded Disability Status Scale (EDSS), presence of comorbidities, presence of gadolinum $(\mathrm{Gd})$-enhancing lesions, presence of more than nine $\mathrm{T} 2$ lesions and of spinal cord lesions on the baseline MRI scan. Comorbidities classification was detailed in a previous paper. ${ }^{4}$

First DMT, date of DMT initiation, date of switch to a new DMT and the new DMT, date of stop and re-start (if the patient re-started the same DMT), and date of last follow-up were recorded. To reduce data complexity, the reason for DMT switch/stop was grouped into two classes: lack of efficacy or intolerance/safety. Treating neurologist made the allocation in one of the two classes. Inefficacy was defined as the occurrence of at least one of the following: 3 month confirmed EDSS progression, relapse occurrence, radiological inefficacy (increase in T2 lesion load, presence of new T1 Gd-enhancing lesions). MRI was collected at baseline and at treatment switch. Spinal cord MRI was not available during follow-up. Relapses were defined following clinical trial criteria: a change in the EDSS with an increase of $\geqslant 0.5$ points on the total score, or an increase of 1 point on two functional systems (FS) or 2 points on one FS, excluding changes involving bowel/bladder or cerebral FS.

When possible, lack of efficacy was further classified as clinical (relapses, EDSS progression) or radiological (MRI lesions). If patients switched therapy for both clinical and radiological lack of efficacy, this was considered as lack of efficacy for clinical reasons. Intolerance/safety was broad including side effects, pregnancy, and John Cunningham Virus (JCV) positivity.

Reasons for DMT switch/stop, EDSS, relapses and MRI data were all entered into local databases before 
data extraction and as part of clinical practice. Neurologists, expert in MS, were in charge of these procedures and performed data extraction as well.

\section{DMTs}

We defined different treatment classes, grouping therapies together based on Italian prescription rules, as first- and second-line therapies. For some analyses, we grouped interferons (IFNs) in one class; we grouped first-line therapies (IFN, glatiramer acetate (GA), teriflunomide (TERI), dymethilfumarate (DMF)), versus second-line therapies (fingolimod and natalizumab) versus other. Also, first-line therapies were grouped as injectables (IFN and GA) with long follow-up versus new orals (TERI and DMF), with shorter follow-up due to their late approval. We defined a horizontal switch as a switch from one to another first-line therapy, and a vertical switch from first- to second-line therapy.

JCV antibody testing was performed as per good clinical practice in natalizumab-treated patients, at least at therapy start and after 12 and 24 infusions, for progressive multifocal leukoencephalopathy (PML) risk stratification. Data on neutralizing antibodies against IFN or natalizumab were not collected as not routinely performed in Italian MS centers.

\section{Statistics}

All statistical analyses were computed using code written in STATA (v.13; StataCorp). Survival analysis was used to generate the Kaplan-Meier estimates for time to switch for any cause. Cumulative incidence analysis accounting for competing risks was based on the model of Fine and Gray ${ }^{27,28}$ and was used to calculate the proportion of patients switching for poor efficacy versus intolerance and for horizontal versus vertical switch. Multivariate Cox regression, adjusted for center, year of diagnosis and age, was used to determine which baseline clinical, radiologic, and demographic features were related to the probability of switching therapy, with different models for switch due to lack of efficacy and intolerance.

Based on the factors emerging from the multivariate models, a baseline score was created to identify patients who start a first-line therapy at a higher risk for an early switch for poor efficacy. The sample was split 50:50 into a training and a validation set. All the analyses run on the training set were then validated on the independent set of data in the validation set. Calibration was performed by comparing the predicted probability of switch with the observed ones plotted as the Kaplan-Meier curves according to the procedure recommended by Royston and Altman. ${ }^{29}$ Harrel $\mathrm{C}$ index was used to assess the discrimination ability of the model. ${ }^{30}$ All the details about the prognostic factors selection, model building, calibration, and discrimination ability of the model are detailed in the appendix. The predictive curves displayed were built on the validation set using the cumulative incidence accounting for competing risks based on the Fine and Gray model. ${ }^{28}$

\section{Results}

\section{Demographics}

We screened 3025 patients; 2954 satisfied the inclusion criteria and were included in the analysis. Recruitment abilities varied among centers, ranging between $10 \%$ and $100 \%$ of all newly diagnosed patients, with a median of $40 \%$.

Demographics and baseline clinical data are shown in Table 1. Follow-up duration had a median of 6.1 years (range 0.1-7.3) for patients with a diagnosis in 2010 and 0.13 years (range $0 .-0.4$ ) for patients with a diagnosis in 2017. Baseline brain MRI data were available for $87 \%$ of patients and spinal cord MRI for $77 \%$. Among 750 patients (31.8\%) with comorbidities, the more frequent were autoimmune diseases $(n=176$; $23.5 \%)$, followed by psychiatric $(n=118 ; 15.7 \%)$, cardiovascular $(n=109 ; 14.5 \%)$, neurologic $(n=77$; $10.2 \%)$, and metabolic $(n=73 ; 9.7 \%)$.

Figure 1 shows the frequency of first therapy choice according to year of diagnosis. Baseline characteristics of patients according to their first therapy are reported in the supplementary material (Table 1S).

Figure 2(a) and (b) shows the overall switch frequency ( $15 \%$ after 1 year, $31 \%$ after 2 years, and $48 \%$ after 3 years), divided into switch for lack of efficacy and switch for intolerance/safety and into horizontal and vertical switch, respectively.

\section{Switch for lack of efficacy}

Multivariate associations between baseline demographic and clinical characteristics and the probability to switch for a perceived lack of efficacy are presented in Table 2. The switch frequency increased with year of diagnosis, with a risk of switch for lack of efficacy that is about fourfold higher in 2016-2017 as compared to 2010-2011 (Table 2). Only licensed therapies were included in this analysis, excluding alemtuzumab since the number of patients treated
Sarah Rasia

Cinzia Cordioli

Multiple Sclerosis Center,

ASST Spedali Civili,

PO di Montichiari (BS),

Montichiari, Italy

Alessia Di Sapio

2nd Neurology Unit and

CRRSM (Regional Referral

Multiple Sclerosis Center),

AOU San Luigi Gonzaga,

Torino, Italy; Regina Monti

Regalis Hospital, Mondovì, Italy

Simona Pontecorvo

Department of Neurology

and Psychiatry, Sapienza

University of Rome, Rome,

Italy

Roberta Grasso

Neurologia Universitaria

OORR, Foggia, Italy

Caterina Barrilà

Department of Neurology,

Valduce Hospital, Como,

Italy 
Table 1. Patients' demographics.

\begin{tabular}{lc} 
Demographic and clinical characteristics & $N=2954$ \\
Age, mean (SD); range & $36(11.6) ; 16-74$ \\
Females, $n$ (\%) & $2(0-8)$ \\
EDSS, median (range) & $376(12.7)$ \\
Year of diagnosis & $416(14.1)$ \\
2010 & $372(12.6)$ \\
2011 & $388(13.1)$ \\
2012 & $438(14.9)$ \\
2013 & $509(17.2)$ \\
2014 & $455(15.4)$ \\
2015 & $3.1(5.4) ; 0.83(0-44.1)$ \\
$2016-2017$ & $3.9(8.7) ; 1.4(0-83.4)$ \\
Years from onset, mean (SD); median (range) & $2.9(0-8)$ \\
Months from diagnosis to first therapy, mean (SD); median (range) & $1(0-5)$ \\
Follow-up from diagnosis, median (range) & $1194 / 2579(46.3)$ \\
Relapses year before baseline, median (range) & $1624 / 2297(70.7)$ \\
Active lesions, $n / N(\%)$ & $1885 / 2625(71.8)$ \\
Spinal cord lesions, $n / N(\%)$ & $750 / 2357(31.8)$ \\
T2 lesions $>9, n / N(\%)$ & \\
Baseline comorbidity, $n / N(\%)$ & \\
\hline SD: standard deviation; EDSS: Expanded Disability Status Scale. & \\
$n / N$ corresponds to the number of patients positive to the considered variable (e.g. active lesions) over the number of patients for \\
whom the presence/absence of the variable is available.
\end{tabular}

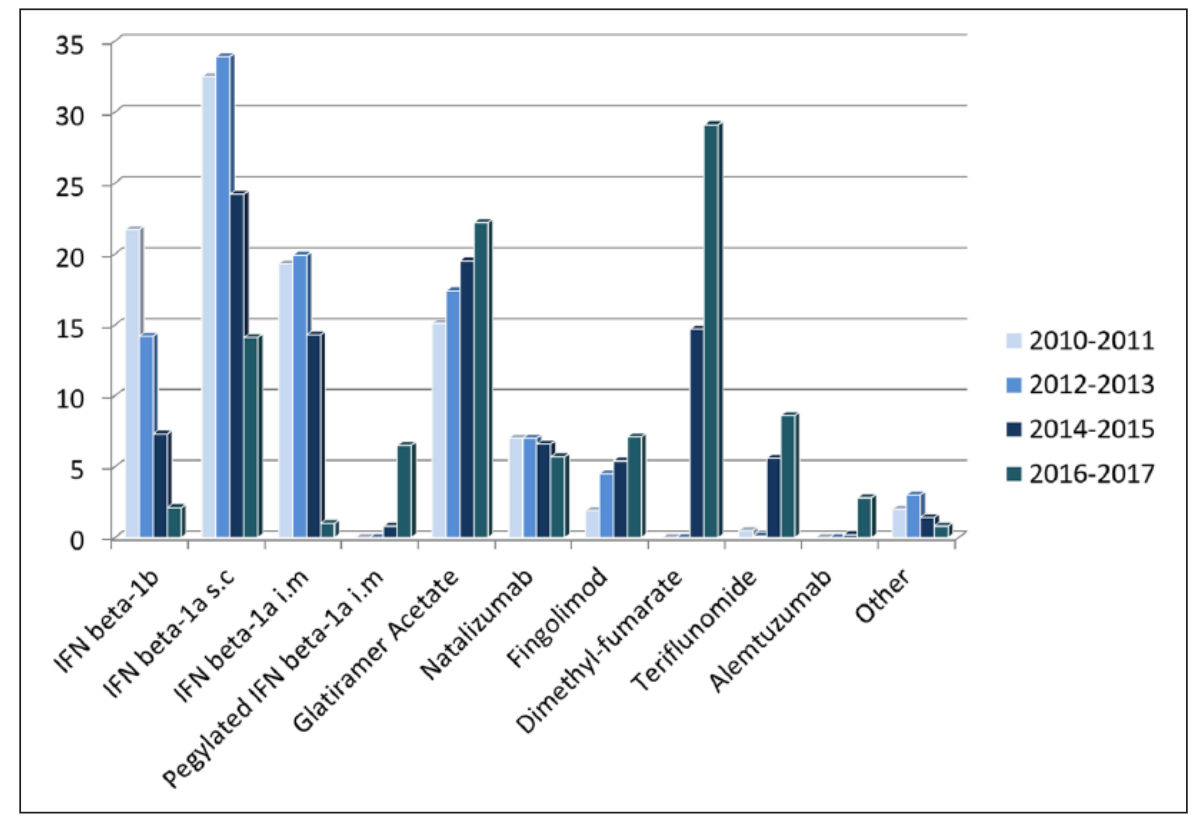

Figure 1. Frequency of first therapy prescription according to year of diagnosis in the whole cohort $(n=2954)$.

with this drug was too low $(n=18)$. Starting with IFN/ GA as compared to oral therapies or a second-line therapy (fingolimod or natalizumab) was the main factor associated with switch probability. The risk of switching (Figure 3(a)) for lack of efficacy is reduced by $50 \%$ starting with fingolimod $(p=0.009)$ and by $87 \%$ starting with natalizumab $(p<0.001)$ as compared to starting with IFN (IFN and GA have a similar 


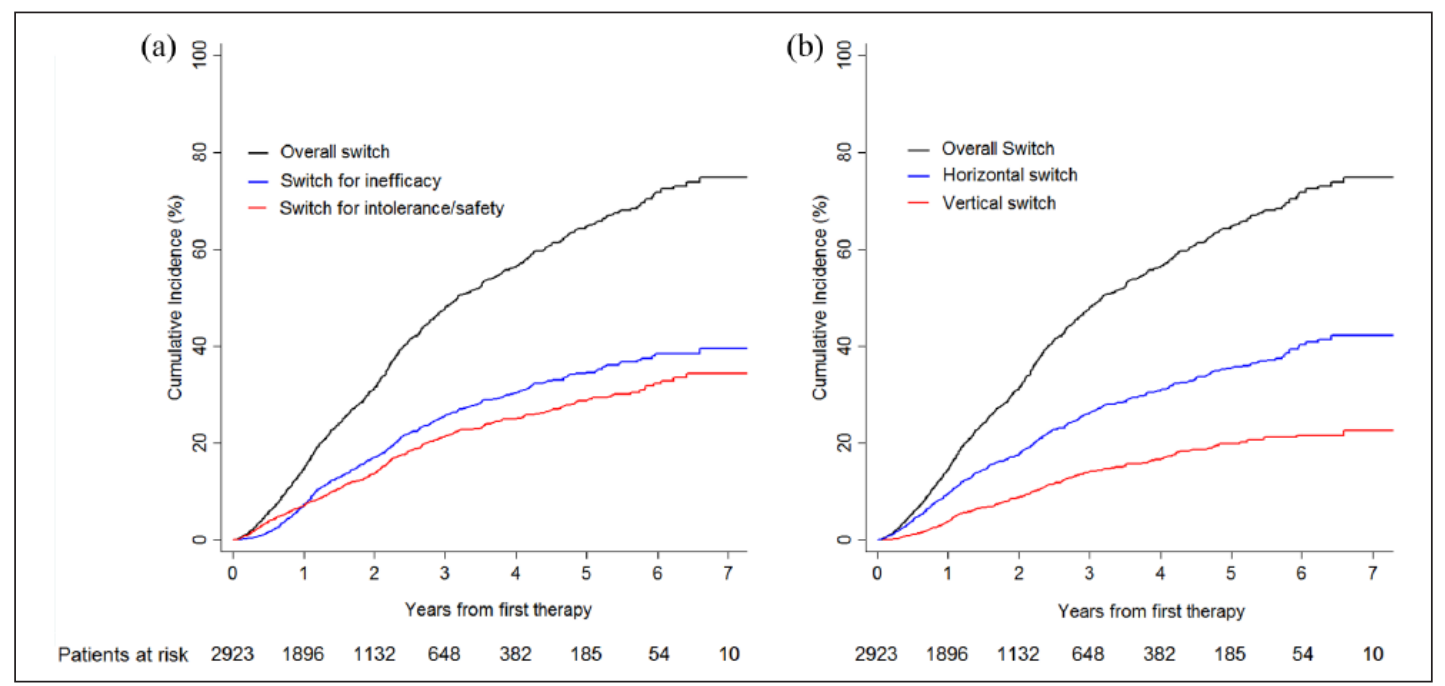

Figure 2. (a) Cumulative incidence of switch for any cause, switch for inefficacy versus switch for intolerance/safety and (b) cumulative incidence of switch for any cause, horizontal versus vertical switch.

risk of switch for lack of efficacy) and is reduced by $40 \%$ starting with DMF $(p=0.037)$ and by $79 \%$ starting with TERI $(p=0.031)$.

Among patients who switched for lack of efficacy $(n=582)$, clinical reasons (relapses or progression of disability) were more frequent $(n=365,67 \%)$, as compared to radiological evidence of activity $(n=182$, $33 \%)$. For 35 patients $(6 \%)$ who switched for lack of efficacy, the reason for the perceived inefficacy was missing. Patients who switched for radiological activity had lower baseline EDSS than those who switched for clinical reasons (1.6 vs 2.1, $p<0.001$ ), and for them horizontal switch was more frequent than vertical switch $(56 \%$ vs $32 \% ; p<0.001)$. The opposite was true in patients who switched for clinical reasons (30\% horizontal vs $60 \%$ vertical).

In order to extract practical guidelines from these results, we tried to identify those patients who started a first-line DMT grouped as IFN/GA (injectables) or DMF/TERI (new orals), and that were at a high risk of an early switch for lack of efficacy. Factors associated with a higher switch probability for lack of efficacy were estimated on the training set $(50 \%$ of the sample) and the discrimination ability of the model was tested on the validation set (Table $2 \mathrm{~S}$, supplementary material). Factors associated with a higher switch probability were age at diagnosis, presence of spinal cord lesions on baseline MRI, a delay between onset and diagnosis and baseline EDSS (Harrel $\mathrm{C}=0.69$ on the training and $\mathrm{C}=0.64$ on the validation set). We created factors associated with a higher risk of early switch and assigned each patient a score between 0 and 4 according to the presence of $0-1,2$, or 3-4 factors. Factors were based on age at diagnosis (younger or older than the median value of 35 years), delay between onset and diagnosis (shorter or longer than the median value of 1 year), and baseline EDSS ( $>$ or $\leqslant 2$; median value; Table $3 \mathrm{~S}$, see online supplementary material). After 2 years, $24 \%$ of patients in IFN/ GA and $16 \%$ of patients in DMF/TERI in the lower risk group ( $\leqslant 1$ risk factors for switch) were estimated to change therapy for lack of efficacy; the percentage was, respectively, $45 \%$ for patients who started with IFN/GA and $30 \%$ for those who started DMF/TERI in the higher risk group ( $>3$ risk factors for switch) (Figure 4).

\section{Switch for intolerance/safety}

Factors associated with intolerance/safety switch are reported in Table 2. In this cohort, 16 patients $(0.5 \%$ of the sample) switched due to reasons related to pregnancy. Different DMTs showed different switch probabilities due to intolerance/safety ( $p$ for heterogeneity $<0.001)$. As shown in Figure 3(b), patients treated with GA, DMF, and fingolimod as their first therapy had the lowest probability to switch due to intolerance/safety. Taking IFN as the reference group, the hazard ratio (HR) for GA was 0.61 (95\% confidence interval $(\mathrm{CI})=0.46-0.81, p=0.001), 0.57$ for DMF $(95 \% \mathrm{CI}=0.35-0.92, p=0.022)$, and 0.35 for fingolimod ( $95 \% \mathrm{CI}=0.19-0.68, p=0.002)$. Patients treated with natalizumab had a higher risk of switching for intolerance/safety $(\mathrm{HR}=1.43,95 \% \mathrm{CI}=1.05-$ $1.94, p=0.022$ ) with a clear switch frequency increase after 2 years (Figure 3(b)), mainly due to positivity to 
Table 2. Multivariate Cox analyses on time to first switch for a perceived inefficacy and for tolerance/safety.

\begin{tabular}{|c|c|c|c|c|}
\hline \multirow{3}{*}{$\begin{array}{l}\text { Demographic and clinical } \\
\text { characteristics }\end{array}$} & \multicolumn{4}{|l|}{ Switch } \\
\hline & \multicolumn{2}{|c|}{ Inefficacy $(n=2851)$} & \multicolumn{2}{|c|}{ Tolerance/safety $(n=2851)$} \\
\hline & $\operatorname{HR}(95 \% \mathrm{CI})^{\mathrm{a}}$ & $p$-value & $\operatorname{HR}(95 \% \mathrm{CI})^{\mathrm{a}}$ & $p$-value \\
\hline \multicolumn{5}{|l|}{ First therapy } \\
\hline IFN $(n=1563)$ & 1.0 (ref) & & 1.0 (ref) & \\
\hline Glatiramer acetate $(n=544)$ & $0.96(0.76-1.21)$ & 0.71 & $0.61(0.46-0.81)$ & 0.001 \\
\hline Fingolimod $(n=139)$ & $0.50(0.29-0.84)$ & 0.009 & $0.35(0.19-0.68)$ & 0.002 \\
\hline Natalizumab $(n=193)$ & $0.13(0.07-0.27)$ & $<0.001$ & $1.43(1.05-1.94)$ & 0.022 \\
\hline Dimethyl-fumarate $(n=307)$ & $0.60(0.37-0.97)$ & 0.037 & $0.57(0.35-0.92)$ & 0.022 \\
\hline Teriflunomide $(n=105)$ & $0.21(0.05-0.87)$ & 0.031 & $0.63(0.30-1.30)$ & 0.21 \\
\hline Age at diagnosis & $0.96(0.95-0.97)$ & $<0.001$ & $1.00(0.99-1.01)$ & 0.73 \\
\hline EDSS, 1-point higher & $1.17(1.07-1.28)$ & 0.001 & $1.07(0.98-1.18)$ & 0.14 \\
\hline \multicolumn{5}{|l|}{ Year of diagnosis } \\
\hline $2010(n=365)$ & 1.0 (ref) & & 1.0 (ref) & \\
\hline $2011(n=409)$ & $0.99(0.76-1.29)$ & 0.93 & $1.16(0.85-1.57)$ & 0.35 \\
\hline $2012(n=356)$ & $1.11(0.84-1.47)$ & 0.46 & $1.25(0.90-1.73)$ & 0.19 \\
\hline $2013(n=377)$ & $1.29(0.96-1.73)$ & 0.086 & $1.51(1.07-2.15)$ & 0.02 \\
\hline $2014(n=426)$ & $1.39(1.01-1.91)$ & 0.041 & $2.00(1.39-2.87)$ & $<0.001$ \\
\hline $2015(n=495)$ & $1.63(1.11-2.40)$ & 0.013 & $3.23(2.15-4.85)$ & $<0.001$ \\
\hline $2016-2017(n=423)$ & $4.06(2.57-6.42)$ & $<0.001$ & $6.23(3.85-10.08)$ & $<0.001$ \\
\hline Diagnosis delay, $\leqslant 1$ year versus $>1$ year & $1.23(1.03-1.47)$ & 0.021 & - & \\
\hline Spinal cord lesions & & & - & \\
\hline No $(n=649)$ & 1.00 (ref) & & & \\
\hline Yes $(n=1549)$ & $1.46(1.18-1.80)$ & 0.001 & & \\
\hline Missing $(n=653)$ & $1.37(0.79-2.40)$ & 0.26 & & \\
\hline Baseline comorbidity & - & & & \\
\hline No $(n=1542)$ & & & 1.00 (ref) & \\
\hline Yes $(n=711)$ & & & $1.28(1.01-1.62)$ & 0.047 \\
\hline Missing $(n=598)$ & & & $0.96(0.43-2.17)$ & 0.93 \\
\hline
\end{tabular}

JCV antibodies test (49 out of $57,86 \%$ ). As previously reported, ${ }^{4}$ the presence of comorbidities at diagnosis was associated with intolerance switch $(\mathrm{HR}=1.28, p=0.047)$.

\section{Discussion}

We report the results from a large multicenter Italian observational study that enrolled newly diagnosed RR-MS patients from 2010 through 2017. AIFA allows fingolimod and natalizumab prescription as first-line therapies in patients with high disease activity at baseline (i.e. two disabling relapses in the previous year and at least one T1 Gd-enhancing lesion or T2 lesion load increase in a recent MRI scan), limiting the prescription of both drugs for treatment-naïve patients. The two new oral therapies, dimethyl-fumarate and teriflunomide, show profound difference with double as much dimethyl-fumarate use as compared to teriflunomide. It is possible that safety issue regarding the possible teriflunomide's teratogen effect may have limited its use in the females within this group of newly diagnosed MS patients, that are, for the vast majority, in the fertile age. A recent report has shown an unexpected safety of teriflunomide in women exposed to the drug during pregnancy ${ }^{31}$ and may lead to a change in future prescription habits.

We found that the availability of new oral therapies reduced the number of patients treated with IFN. The effect was more evident for IFN beta- $1 \mathrm{~b}$, probably due to its more frequent administration, and troublesome adherence. The decrease of intramuscular (i.m.) IFN beta-1a can be partly explained by 


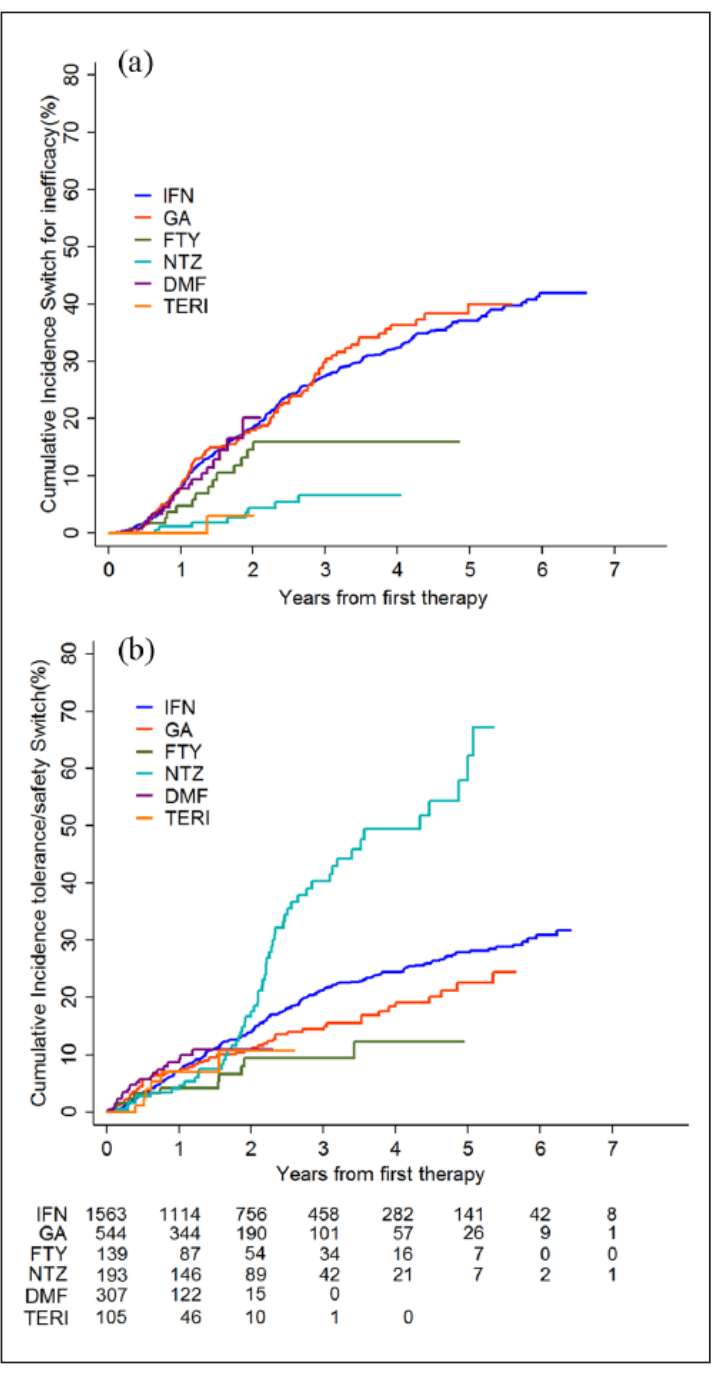

Figure 3. Cumulative incidence of switch for inefficacy (a) and of switch for intolerance/safety (b) according to first therapy choice.

the introduction of the pegylated version of this IFN. The use of GA remained stable over time, with a small increase in prescriptions in the last years. Its different mechanisms of action, tolerability profile and use in patients with comorbidities may have contributed to an increase in prescriptions. Fingolimod was approved at the end of 2011, and its prescription rate increased progressively. Natalizumab use showed a small decline over time. Alemtuzumab was approved in Italy in 2015 and its use as a first therapy started to increase in 2016.

Overall, our study shows that poor efficacy is the predominant cause of switch from first-line therapy as compared to safety/intolerance. This indicates that the concerns raised by the probability of disability progression supersede those secondary to safety/intolerance.
Alternatively, DMTs currently in use in MS are overall well tolerated and side effects necessitating a therapeutic change are much more rare than clinical or radiological activity. Poor efficacy switch was more frequent in patients treated with first-line injectable therapies as compared to second-line treatments.

With the first therapy being equal, we found the following factors to be predictors of DMT switch due to poor efficacy: younger age, reduced delay between onset and diagnosis, positive spinal MRI and higher EDSS. While the first three factors may indicate a more aggressive disease, higher EDSS as a predictive factor for poor efficacy switch may lead to different interpretations. Intuitively, a higher EDSS may be linked to a more aggressive disease, increasing the likelihood of switching for poor efficacy. A second possible explanation is that neurologists are more concerned about disease progression in patients with higher EDSS scores, as this may lead to irreversible disability, and are more willing to change therapy. This is confirmed by the higher prevalence of patients with higher EDSS and vertical (i.e. more effective) switches in those switching for clinical poor efficacy as compared to radiological poor efficacy. The higher prevalence of horizontal switches in patients with radiological poor efficacy supports this theory, as this may be translated into a search for a new mechanism of action rather than an increase in overall efficacy.

Using baseline factors to identify high-risk patients proved to be useful as it correctly identified subjects with a high switch propensity. Results showed that IFN/GA patients with at least three of the four defined risk factors for poor efficacy switch (younger age, higher EDSS, presence of spinal cord lesions, shorter delay between onset and diagnosis) are at a higher risk $(>30 \%)$ to switch for poor efficacy within 2 years. A validation on an external dataset would be warranted to generalize the prediction ability of the proposed model. We could speculate that treating high-risk patients with second-line DMTs as a first choice may help reduce poor efficacy switches and possibly have a better control on disease progression. In more recent years, switch tendency increased, and this may be the result of a larger therapeutic repertoire, thus encouraging earlier DMT switch for perceived incomplete efficacy.

As for intolerance/safety switch, the choice of first treatment played a different role in DMT switch. GA and fingolimod showed the best persistence on treatment. This is supported by the safety/tolerance profile of both DMTs. Natalizumab had very few switches until the 2-year cut point when the majority of patients 


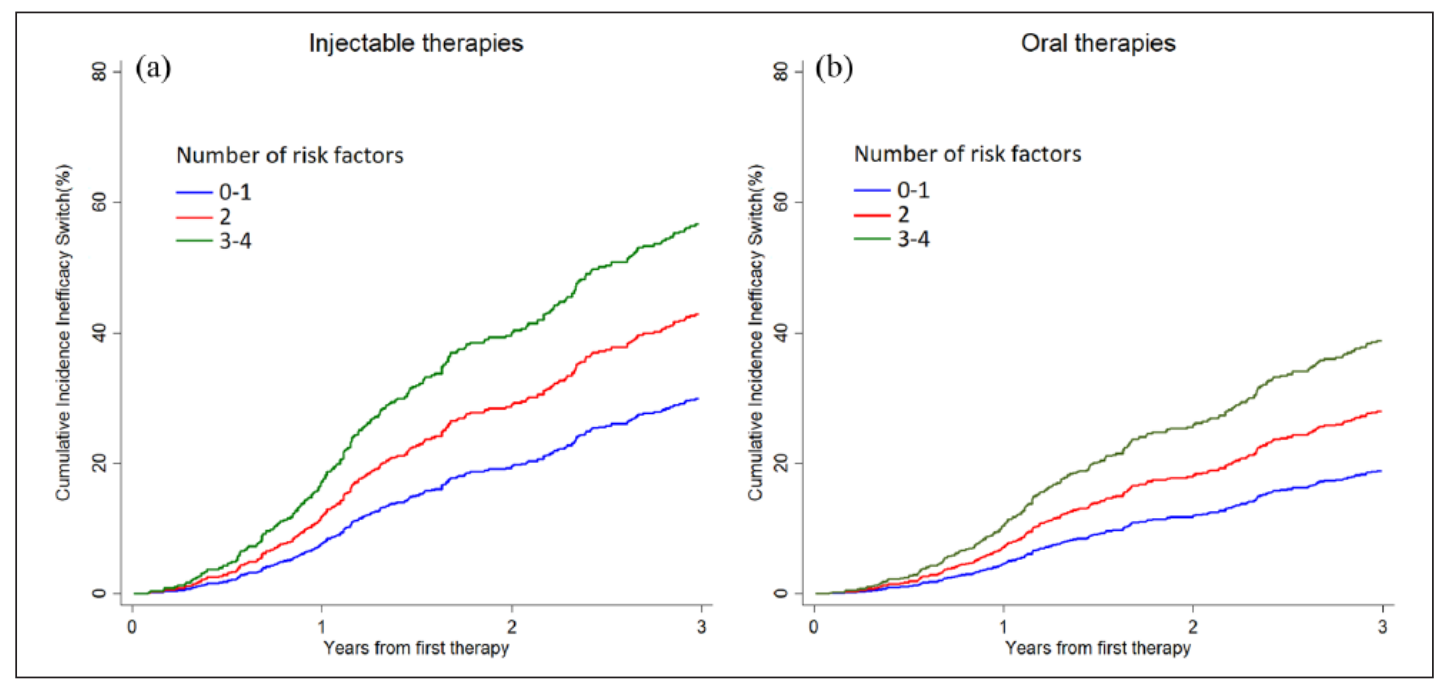

Figure 4. Estimated cumulative incidence of switch for inefficacy in patients who started a first-line therapy with injectable therapies (interferon or glatiramer acetate) (a) or with oral therapies (dimethyl-fumarate or teriflunomide) (b) according to the number of baseline risk factors (age younger than 35 years, baseline EDSS higher than 2, presence of spinal cord lesions, delay between onset and diagnosis lower than 1 year).

were switched to other drugs, mainly because of positive anti-JCV antibodies. We are aware that the high number of intolerance/safety switches in the natalizumab group may have masked a discrete number of inefficacy switches leading to its underestimation. New oral drugs showed tolerability comparable to injectable first-line drugs with a lower rate of switch for lack of efficacy.

Our study suffers from several limitations due to the observational and retrospective nature of the design that does not allow to fully control for possible heterogeneities in patient assessment criteria and data collection procedures across centers. On the other hand, the centers included in this study were all highly specialized MS centers and the adjustment in the analyses for a center effect can limit the potential influence of such heterogeneities.

In contrast to our results, recent papers pointing at the persistence on injectable or oral first-line therapies ${ }^{32,33}$ showed that in the short term, poor tolerance was the main determinant of therapy switch. These studies, however, had different inclusion criteria, considering patients with previous DMT history and excluding patients escalating to a second-line therapy. In contrast, our study was focused on treatment-naïve patients and evaluated all possible causes for treatment switch.

In conclusion, our data showed that almost half of patients had their first treatment changed, mainly for poor efficacy, after 3 years from treatment start. We also show that identification of high-risk patients may be helpful in first treatment choice so to concentrate available resources in patient follow-up. The advent of new oral therapies and new monoclonal antibodies will hopefully improve DMT efficacy and patients persistence.

\section{Acknowledgements}

The authors wish to thank Novartis Pharma for supporting the meetings of the iMUST group. F.S. acquired the data, participated in data analysis and interpretation, and wrote the manuscript draft; R.L. acquired the data, participated in data analysis and interpretation, and wrote the manuscript draft; A.S. managed, analyzed, and interpreted the data and wrote the manuscript draft; G.T.M., E.S., S.L., A.R., P.A., D.B., M.C., E.B., R.C., G.M., S.B., L.L., I.R.Z., A.L., S.R., P.G.L., S.L., B.F., V.B., J.F., E.C., G.F., V.T.C., A.S., S.R., C.C., A.D., S.P., R.G., C.B., C.V.R., S.E., D.I., F.B., F.B. acquired the data, participated in data analysis and interpretation, and contributed to manuscript draft; M.P.S. analyzed and interpreted the data, wrote the manuscript draft and supervised the study; F.S. and R.L. equally contributed to the work.

\section{Declaration of Conflicting Interests}

The author(s) declared the following potential conflicts of interest with respect to the research, authorship, and/ or publication of this article: F.S. received personal compensation from Novartis, Almirall, Genzyme, Biogen, Merck Serono Forward Pharma, and TEVA for public speaking, editorial work, and advisory boards. R.L. received personal compensation from Merck Serono, Biogen, Novartis, Almirall, Genzyme, and 
TEVA for public speaking, editorial work, and advisory boards. A.S. received teaching honoraria from Novartis. G.T.M. received personal compensation from Serono, Biogen, and TEVA for public speaking and advisory boards. P.A. served as advisor and received speaking honoraria from Novartis, Merck Serono, Genzyme, Biogen, and Teva Italia. D.B. received honoraria from Almirall for the creation of editorial publications, and travel grants for participation to international congresses from Genzyme and TEVA. L.P. received consulting fees from Biogen and Novartis; speaker honoraria from Biogen, Genzyme, Novartis, and Teva; travel grants from Biogen, Genzyme, Novartis, and Teva; research grants from Genzyme. E.B. has nothing to disclose. S.L.F. received funding for travel and for advisory board from Genzyme, Biogen Idec, Teva, Merck Serono. A.R. received personal compensation from Biogen Idec, Genzyme, Novartis, and Merck Serono for public speaking and advisory boards. M.C. received personal compensation for participating to advisory boards by Merck Serono and Biogen; travel expenses for congresses paid by Merck, Biogen, Novartis, and Genzyme. S.B. received speaker honoraria from Merck Serono, Novartis, Teva, and Genzyme; Advisory Board honoraria from Teva, Novartis, Biogen. S.L.G. has nothing to disclose. S.R. acted as an Advisory Board member of Biogen Idec, Bayer Schering, Merck Serono, Teva, Novartis, and Genzyme and received funding for traveling and honoraria for speaking or writing from Biogen Idec, Merck Serono, Teva, Novartis, Bayer Schering, Genzyme, Almirall. She received support for research project by Teva, Merck Serono, and Bayer Schering and is involved as principal investigator in clinical trials for Teva and Roche. A.L. has received personal compensation from Novartis, Genzyme, Biogen, and TEVA for public speaking and advisory boards. J.F. serves on scientific advisory boards for Biogen, received honoraria for speaking from Merck Serono, Biogen, and Teva, and received a research grant from Merck Serono. E.C. received personal compensation from Almirall, Bayer, Biogen, Genzyme, Novartis, Serono, and TEVA for public speaking, editorial work, and advisory boards. V.T.C. received personal compensation from Novartis, Almirall, Genzyme, and Teva for public speaking, editorial work, and advisory boards. I.R.Z. has served on a scientific advisory board for Biogen Idec and received funding for travel and/or speaker honoraria from Genzyme, Biogen Idec, Teva, Merck, and Novartis. A.S. has received funding for travel and/or speaker honoraria from Novartis, Teva, Merck Serono, and Genzyme. E.S. received personal compensation from Almirall, Biogen, Genzyme, Novartis, and Teva for traveling and advisory boards. S.R. has nothing to disclose. C.C. received personal compensations for consultanting from Merck Serono and Novartis. R.C. received funding for travel and/or speaker honoraria from Genzyme, Biogen Idec, Teva, Merck Serono, and Novartis. S.P. received personal compensation from Almirall, Biogen, Genzyme, and Teva for public speaking and advisory boards. A.D.S. received personal compensation from Novartis, Biogen, Merck Serono, Teva, and Bayer Schering for public speaking and advisory boards; received funding for travel/meetings from Merck Serono, Biogen, Novartis, Genzyme, Allergan, and Medtronic. L.L. received funding for travel and/or speaker honoraria from Novartis, Genzyme, Teva, Merck, Almirall, and Bayer. C.B. has nothing to disclose. C.V.R. has nothing to disclose. B.F. has nothing to disclose. S.E. has nothing to disclose. D.I. has nothing to disclose. F.G. received teaching fees from Novartis. M.P.S. received personal compensation for consulting services and for speaking activities from Merck Serono, Teva, Novartis, Roche, Genzyme, and Biogen.

\section{Funding}

The author(s) disclosed receipt of the following financial support for the research, authorship, and/or publication of this article: Novartis Pharma was not involved in this project and did not have any access to the data.

\section{ORCID iD}

Damiano Baroncini (iD https://orcid.org/0000-00034234-8956

\section{References}

1. European Medicines Agency (EMA). Tysabri: EPAR product information, 13 October 2016.

2. European Medicines Agency (EMA). Gilenya: EPAR product information, 21 December 2016.

3. Zhang $\mathrm{T}$, Tremlett $\mathrm{H}$, Leung $\mathrm{S}$, et al. Examining the effects of comorbidities on disease-modifying therapy use in multiple sclerosis. Neurology 2016; 86: $1287-1295$.

4. Laroni A, Signori A, Maniscalco GT, et al. Assessing association of comorbidities with treatment choice and persistence in MS: A real-life multicenter study. Neurology 2017; 89: 2222-2229.

5. D'Amico E, Leone C, Caserta C, et al. Oral drugs in multiple sclerosis therapy: An overview and a critical appraisal. Expert Rev Neurother 2015; 15: 803-824.

6. European Medicines Agency (EMA). Lemtrada: EPAR product information, 7 July 2016.

7. European Medicines Agency (EMA)/Committee for Medicinal Products for Human Use (CHMP). Ocrevus-Initial authorization, 10 November 2017. 
8. Kalincik T, Horakova D, Spelman T, et al. Switch to natalizumab versus fingolimod in active relapsingremitting multiple sclerosis. Ann Neurol 2015; 77 : 425-435.

9. Prosperini L, Saccà $\mathrm{F}$, Cordioli $\mathrm{C}$, et al. Realworld effectiveness of natalizumab and fingolimod compared with self-injectable drugs in nonresponders and in treatment-naïve patients with multiple sclerosis. J Neurol 2017; 264(2): 284-294.

10. Lanzillo R, Carotenuto A, Moccia M, et al. A longitudinal real-life comparison study of natalizumab and fingolimod. Acta Neurol Scand 2016; 136: 217-222.

11. Koch-Henriksen N, Magyari M, Sellebjerg F, et al. A comparison of multiple sclerosis clinical disease activity between patients treated with natalizumab and fingolimod. Mult Scler 2016; 23: 234-241.

12. Barbin L, Rousseau C, Jousset N, et al. Comparative efficacy of fingolimod vs natalizumab: A French multicenter observational study. Neurology 2016; 86(8): 771-778.

13. Baroncini D, Ghezzi A, Annovazzi PO, et al Natalizumab versus fingolimod in patients with relapsing-remitting multiple sclerosis non-responding to first-line injectable therapies. Mult Scler 2016; 22(10): 1315-1326.

14. Lanzillo R, Moccia M, Laplaud DA, et al. Comparative efficacy of fingolimod vs natalizumab: A French multicenter observational study. Neurology 2016; 87(10): 1066.

15. Havrdova E, Galetta S, Stefoski D, et al. Freedom from disease activity in multiple sclerosis. Neurology 2010; 74(Suppl. 3): S3-S7.

16. Gajofatto A and Benedetti MD. Treatment strategies for multiple sclerosis: When to start, when to change, when to stop? World J Clin Cases 2015; 3: 545-555.

17. D'Amico E, Leone $\mathrm{C}$, Zanghì $\mathrm{A}$, et al. Lateral and escalation therapy in relapsing-remitting multiple sclerosis: A comparative study. J Neurol 2016; 263 : 1802-1809.

18. Lanzillo R, Bonavita S, Quarantelli M, et al. Natalizumab is effective in multiple sclerosis patients switching from other disease modifying therapies in clinical practice. Neurol Sci 2013; 34: 521-528.

19. Lugaresi A, De Robertis F, Clerico M, et al. Longterm adherence of patients with relapsing-remitting multiple sclerosis to subcutaneous self-injections of interferon $\beta$-1a using an electronic device: The RIVER study. Expert Opin Drug Deliv 2016; 13: 931-935.

Visit SAGE journals online journals.sagepub.com/ home/msj

(SAGEjournals
21. Paolicelli D, Cocco E, Di Lecce V, et al. Exploratory analysis of predictors of patient adherence to subcutaneous interferon beta-1a in multiple sclerosis: TRACER study. Expert Opin Drug Deliv 2016; 13: 799-805.

22. Warrender-Sparkes M, Spelman T, Izquierdo G, et al. The effect of oral immunomodulatory therapy on treatment uptake and persistence in multiple sclerosis. Mult Scler 2016; 22: 520-532.

23. Moccia M, Palladino R, Carotenuto A, et al. Predictors of long-term interferon discontinuation in newly diagnosed relapsing multiple sclerosis. Mult Scler Relat Disord 2016; 10: 90-96.

24. Meyniel C, Spelman T, Jokubaitis VG, et al. Country, sex, EDSS change and therapy choice independently predict treatment discontinuation in multiple sclerosis and clinically isolated syndrome. PLOS ONE 2012; 7: e38661.

25. McDonald WI, Compston A, Edan G, et al. Recommended diagnostic criteria for multiple sclerosis: Guidelines from the International Panel on the diagnosis of multiple sclerosis. Ann Neurol 2001; 50: $121-127$.

26. Polman $\mathrm{CH}$, Reingold SC, Banwell B, et al. Diagnostic criteria for multiple sclerosis: 2010 revisions to the McDonald criteria. Ann Neurol. 2011; 69: 292-302.

27. Coviello V and Boggess M. Cumulative incidence estimation in the presence of competing risks. Stata $J$ 2004; 4: 103-112.

28. Andersen PK, Geskus RB, Witte T, et al. Competing risks in epidemiology: Possibilities and pitfalls. Int $J$ Epidemiol 2012; 41: 861-870.

29. Royston P and Altman DG. External validation of a Cox prognostic model: Principles and methods. BMC Med Res Methdol 2013; 13: 33.

30. Steyerberg EW, Vickers AJ, Cook NR, et al. Assessing the performance of prediction models: A framework for some traditional and novel measures. Epidemiology 2010; 21: 128-138.

31. Davenport L, Beyer B, Truffinet P, et al. Nonclinical data demonstrate high sensitivity of rats versus humans to embryo-foetal toxicity when exposed to teriflunomide. Mult Scler 2016; 22(S3): 145516.

32. Ferraro D, Camera V, Baldi E, et al. First-line diseasemodifying drugs in relapsing-remitting multiple sclerosis: An Italian real-life multicenter study on persistence. Curr Med Res Opin 2018; 12: 1-5.

33. Lanzillo R, Prosperini L, Gasperini C, et al; R.I.Re. MS study group. A multicentRE observational analysiS of PErsistenCe to Treatment in the new Multiple Sclerosis era: The RESPECT study. $J$ Neurol 2018; 265: 1174-1183. 